\title{
Spin squeezing criterion with local unitary invariance
}

\author{
A. R. Usha Devi ${ }^{1,2}$, Xiaoguang Wang ${ }^{1}$, and B. C. Sanders ${ }^{1}$ \\ 1. Department of Physics and Australian Centre for Quantum Computer Technology, \\ Macquarie University, Sydney, New South Wales 2109, Australia. and \\ 2. Department of Physics, Bangalore University, \\ Jnanabharathi Campus, Bangalore 560 056, India.
}

(Dated: October 29, 2018)

\begin{abstract}
We propose a spin squeezing criterion for arbitrary multi-qubit states that is invariant under local unitary operations. We find that, for arbitrary pure two-qubit states, spin squeezing is equivalent to entanglement, and multi-qubit states are entangled if this new spin squeezing parameter is less than 1.

PACS numbers: 03.67.-a, 03.65.Ud
\end{abstract}

\section{INTRODUCTION}

The non-classical nature of quantum entanglement is a key ingredient in the rapidly developing science of quantum information [1, 2]. One of the important tasks involved is quantifying quantum entanglement, which is essential in assessing the performance of a quantum system in several applications such as quantum teleportation [3], quantum cryptography [4, [5, [6], quantum computation [7, 8, 9, 10] and quantum communication 10, 11]. There has been an ongoing effort devoted to characterizing quantum entanglement, especially the entanglement of multi-particle states shared by several distant parties 12, 13, 14, 15, 16, 17, 18, 19, 20]. In view of the recent interest in creating and manipulating correlated collective atomic states 21, 22, 23, 24, 25, 26, 27, 28, 29], an experimentally relevant characterization of entanglement in terms of spin squeezing $21,30,31,32,33,34,35,36,37,38,39,40,41,42,43$ has been highlighted [29, 44]. Most studies are concentrated on collective symmetric multi-particle states where individual particles are assumed to be inaccessible. Our aim is to develop a spin squeezing criterion for a multiqubit system that is invariant under local unitary transformations on individual qubits. The motivation for this work is to explore the fundamental connection between spin squeezing and entanglement, recognizing that entanglement is invariant under local unitary transformation.

Let us first review spin squeezing criteria. Several definitions of spin squeezed states have been proposed in the literature 29, 30, 45, 46, 47]. Kitagawa and Ueda 30] pointed out that a definition of spin squeezing, based only on the uncertainty relation [45], does not reveal quantum correlations among the elementary spins. They first identified a mean spin direction

$$
\hat{n}_{0}=\frac{\langle\vec{J}\rangle}{|\langle\vec{J}\rangle|}, \quad|\langle\vec{J}\rangle|=\sqrt{\langle\vec{J}\rangle \cdot\langle\vec{J}\rangle},
$$

where the collective spin operator $\vec{J}$ for an $N$-qubit system is defined by $\vec{J} \equiv \frac{1}{2} \sum_{i=1}^{N} \vec{\sigma}_{i}$ with $\vec{\sigma}_{i}=\left(\sigma_{x}, \sigma_{y}, \sigma_{z}\right)^{T}$ the Pauli vector of the $i^{\text {th }}$ qubit. Associating a mutually orthonormal set $\left(\hat{n}_{\perp}, \hat{n}_{\vdash}, \hat{n}_{0}\right)$, we have another set of collective operators

$$
J_{\mu}=\vec{J} \cdot \hat{n}_{\mu}, \mu \in\{\perp, \vdash, 0\},
$$

which satisfy the angular momentum commutation relations. Kitagawa and Ueda [30] proposed that a multiqubit state can be regarded as spin squeezed if the minimum of $\Delta J_{\theta}$ of a spin component normal to the mean spin direction is smaller than the standard quantum limit $\sqrt{N} / 2$ of the spin coherent state, where

$$
J_{\theta}=J_{\perp} \cos \theta+J_{\vdash} \sin \theta .
$$

A parameter incorporating this feature may be defined by

$$
\xi_{1} \equiv \frac{2\left(\Delta J_{\theta}\right)_{\min }}{\sqrt{N}}
$$

where the minimization is over $\theta$. A spin squeezed state satisfies $\xi_{1}<1$.

In the context of Ramsey spectroscopy on a sample of $N$ two-level atoms, Wineland et al. 47] showed that the frequency resolution depends on the parameter

$$
\xi_{2}=\frac{\sqrt{N}\left(\Delta J_{\theta}\right)_{\min }}{\left\langle J_{0}\right\rangle}=\frac{2 N \xi_{1}}{\left\langle J_{0}\right\rangle},
$$

and spin squeezing manifested by $\xi_{2}<1$ leads to reduction in the frequency noise. This identification opened up possible applications of spin squeezed states to high precision atomic clocks [4] and atomic interferometers [4]. Note that spin squeezing established through $\xi_{1}<1$ is a necessary condition for $\xi_{2}<1$, but it is not a sufficient condition since $\left\langle J_{0}\right\rangle \leq 2 N$.

Spin squeezing, in the original sense, is defined for multi-qubit states belonging to the maximum multiplicity subspace of the collective angular momentum operator $\vec{J}$. These states exhibit symmetry under the interchange of particles. The concept of spin squeezing is therefore restricted to symmetric multi-particle systems that are accessible to collective operations alone. 
We explore the possibility of extending the concept of spin squeezing to multi-qubit systems, where individual qubits are accessible. This requires a criterion of spin squeezing that exhibits invariance under local unitary operations on the qubits. In contrast, Eqs. (4) and (5) are not invariant under arbitrary local unitary transformations on the qubits. In order to see this, consider a state of two qubits given by

$$
\left|\Psi_{12}\right\rangle=\cos \phi|01\rangle+\sin \phi|10\rangle,
$$

in which the expectation value of the collective angular momentum $\langle\vec{J}\rangle=0$, and therefore one cannot properly define spin squeezing for this state. However, under a local unitary operation $U_{1} \otimes U_{2}=\mathbb{1} \otimes \sigma_{x}$, the state $\left|\Psi_{12}\right\rangle$ transforms to

$$
\left|\Psi_{12}^{\prime}\right\rangle=\cos \phi|00\rangle+\sin \phi|11\rangle,
$$

where $\mathbb{1}$ is a $2 \times 2$ identity matrix. One can readily verify that the state $\left|\Psi_{12}^{\prime}\right\rangle$ is spin squeezed with the squeezing parameters of Eqs. (4) and (15) given by

$$
\xi_{1}=\sqrt{1-|\sin 2 \phi|} \leq 1, \quad \xi_{2}=\frac{1}{\sqrt{1+|\sin 2 \phi|}} \leq 1
$$

Therefore, the spin squeezing by either criterion is modified by a local unitary transformation.

A simpler example is a product state $\left|\psi_{1}\right\rangle\left|\psi_{2}\right\rangle$, which is in general non-symmetric. For the non-symmetric case, we find that the squeezing parameter $\xi_{1}$ can be less than 1 . This fact implies that the squeezing parameter $\xi_{1}$ works well for symmetric states; however, for non-symmetric cases, one cannot distinguish a correlated state from an uncorrelated one.

In this paper, we provide a local unitary invariant version of spin squeezing criteria, which reveals quantum correlations for arbitrary multi-qubit states. It is wellknown that quantum entanglement is locally unitary invariant. The local unitary invariant property of the spin squeezing criteria and quantum entanglement suggests that they may exhibit closer relations comparing with relations between the original spin squeezing criteria and entanglement.

The paper is organized as follows: In Sec. II, we introduce two spin squeezing criteria, which are shown to be locally unitary invariant. In Sec. III, we provide relations between spin squeezing and quantum entanglement, and find that (i) for arbitrary two-qubit pure states, spin squeezing implies entanglement, and vice versa, and (ii) for an arbitrary multi-qubit state, if the local invariant version of squeezing parameter $\xi_{2}$ is less than 1 , then the state is entangled. The conclusion is given in Sec. IV.

\section{LOCAL UNITARY INVARIANT SPIN SQUEEZING CRITERIA}

Now we introduce local unitary invariant spin squeezing criteria for $N$ qubits. We denote unit vectors along the mean orientations (mean spin directions) of the qubit $i$ by

$$
\hat{n}_{i 0}=\frac{\left\langle\vec{\sigma}_{i}\right\rangle}{\left|\left\langle\vec{\sigma}_{i}\right\rangle\right|}, \quad\left|\left\langle\vec{\sigma}_{i}\right\rangle\right|=\left(\left\langle\vec{\sigma}_{i}\right\rangle \cdot\left\langle\vec{\sigma}_{i}\right\rangle\right)^{\frac{1}{2}}
$$

Associating a mutually orthogonal set $\left(\hat{n}_{i \perp}, \hat{n}_{i \vdash}, \hat{n}_{i 0}\right)$ of unit vectors with each qubit, we may define the collective operators

$$
\perp=\frac{1}{2} \sum_{i=1}^{N} \vec{\sigma}_{i} \cdot \hat{n}_{i \perp, \vdash}=\frac{1}{2} \sum_{i=1}^{N} \vec{\sigma}_{i} \cdot \hat{n}_{i \vdash, 0}=\frac{1}{2} \sum_{i=1}^{N} \vec{\sigma}_{i} \cdot \hat{n}_{i 0},
$$

which satisfy the usual angular momentum commutation relations. For instance,

$$
[\perp, \vdash]=\frac{\mathrm{i}}{2} \sum_{i=1}^{N} \vec{\sigma}_{i} \cdot \hat{n}_{i 0}=\mathrm{i}_{0}
$$

and this leads to the uncertainty relations

$$
\left(\Delta_{\perp}\right)\left(\Delta_{\vdash}\right) \geq \frac{1}{2}\langle 0\rangle .
$$

Analogous to the definitions of $\xi_{k}, k \in\{1,2\}$, we define the two spin squeezing parameters $\tilde{\xi}_{k}$,

$$
\tilde{\xi}_{1}=\frac{2\left(\Delta_{\left\{\theta_{i}\right\}}\right)_{\min }}{\sqrt{N}}, \quad \tilde{\xi}_{2}=\frac{\sqrt{N}\left(\Delta_{\left\{\theta_{i}\right\}}\right)_{\min }}{\langle 0\rangle}
$$

where

$$
\left\{\theta_{i}\right\}=\frac{1}{2} \sum_{i=1}^{N} \vec{\sigma}_{i} \cdot \hat{n}_{\theta_{i}}, \hat{n}_{\theta_{i}}=\hat{n}_{i \perp} \cos \theta_{i}+\hat{n}_{i \vdash} \sin \theta_{i},
$$

and the minimization is over all $\theta_{i}, i \in\{1,2, \ldots, N\}$. They satisfy local unitary invariance as we show below. Therefore, we have given locally invariant versions of the original spin squeezing criteria. A system of $N$ qubits are regarded as spin squeezed if $\tilde{\xi}_{k}<1$ for $k \in\{1,2\}$.

Now we show the key property of the spin squeezing parameter $\tilde{\xi}_{k}$ given by

Proposition 1: The spin squeezing parameters $\tilde{\xi}_{k}$ are invariant under the local unitary operator

$$
U=U_{1} \otimes U_{2} \otimes \cdots \otimes U_{N}
$$

Proof: From the expressions of $\tilde{\xi}_{k}$ given by Eq. (13), we only need to prove that $\left\langle\mathcal{J}_{0}\right\rangle$ and $\left(\Delta_{\left\{\theta_{i}\right\}}\right)_{\text {min }}^{2}$ are invariant. We now use the well-known fact that, for every unitary transformation $U_{i}$ on a qubit $i$, there corresponds a unique $3 \times 3$ orthogonal rotation matrix $O_{i}$ such that

$$
\vec{\sigma}_{i}^{\prime} \equiv U_{i}^{\dagger} \vec{\sigma}_{i} U_{i}=O_{i} \vec{\sigma}_{i}, \quad\left\langle\vec{\sigma}_{i}^{\prime}\right\rangle=O_{i}\left\langle\vec{\sigma}_{i}\right\rangle .
$$

From Eqs. (9) and (10), we may write the expectation value $\left\langle\mathcal{J}_{0}\right\rangle$ as

$$
\left\langle\mathcal{J}_{0}\right\rangle=\frac{1}{2} \sum_{i=1}^{N}\left|\left\langle\vec{\sigma}_{i}\right\rangle\right|
$$


From Eqs. (16) and (17), we observe that the expectation value $\left\langle\mathcal{J}_{0}\right\rangle$ is invariant under the local unitary transformation.

To prove that $\left(\Delta_{\left\{\theta_{i}\right\}}\right)_{\min }^{2}$ is invariant, we first write it as

$$
\left(\Delta_{\left\{\theta_{i}\right\}}\right)_{\min }^{2}=\frac{1}{4}\left[N+2\left(\sum_{i=1}^{N} \sum_{j>i=1}^{N} \hat{n}_{\theta_{i}}^{T} \mathcal{T}^{(i j)} \hat{n}_{\theta_{j}}\right)_{\min }\right],
$$

where

$$
\mathcal{T}_{\alpha \beta}^{(i j)}=\left\langle\sigma_{i \alpha} \sigma_{j \beta}\right\rangle, \quad \alpha, \beta=x, y, z,
$$

are the matrix elements of the $3 \times 3$ correlation matrix [49] for a given qubit pair $i, j$. Note that $\hat{n}_{\theta_{i}}$ is a column vector.

From Eq. (16), under the unitary transformations $U$, the parameter $\hat{n}_{\theta_{i}}$ and the correlation matrix $\mathcal{T}^{(i j)}$ transform as follows [49]:

$$
\hat{n}_{\theta_{i}}^{\prime}=O_{i} \hat{n}_{\theta_{i}}, \quad \mathcal{T}^{\prime(i j)}=O_{i} \mathcal{T}^{(i j)} O_{j}^{T} .
$$

Applying the above equation to Eq. (18) leads to the invariance of $\left(\Delta_{\left\{\theta_{i}\right\}}\right)_{\min }^{2}$ under the unitary transformation $U$.

Though the minimization $\left(\Delta_{\left\{\theta_{i}\right\}}\right)_{\min }$ over all the directions $\hat{n}_{\theta_{i}}$ (orthogonal to the qubit orientations) appears to be non-trivial, local invariance of $\tilde{\xi}_{k}$ leads to a simplified analysis for multi-qubit systems. For instance, we may consider a product state with each qubit being in a different state. For this case, we can always find a unitary operator $U$ to transform this state to the symmetric product state $|0\rangle \otimes \cdots \otimes|0\rangle$, for which the squeezing parameters $\tilde{\xi}_{k}$ are easily found to be 1 . This simple example indeed displays that when the quantum correlations do not exist, the spin squeezing parameter cannot be less than 1 . Recognizing that the both the spin squeezing parameter $\tilde{\xi}_{k}$ and quantum entanglement are invariant under local unitary transformations, we next investigate the relations between spin squeezing and entanglement.

\section{RELATIONS TO QUANTUM ENTANGLEMENT}

Spin squeezing is closely related to, and implies, quantum entanglement [29, 50, 51, 52, 53]. As the spin squeezing criteria we propose satisfy local unitary invariance, a close relation between these criteria and quantum entanglement is expected.

\section{A. Two-qubit states}

To see the relations of the local unitary invariant spin squeezing to quantum entanglement, let us examine the arbitrary two-qubit pure state given by

$$
\left|\Phi_{12}\right\rangle=\alpha|00\rangle+\beta|01\rangle+\gamma|10\rangle+\delta|11\rangle .
$$

It has been shown earlier [50] that for a pure symmetric state of pair qubits (with $\beta=\gamma$ in Eq. (21)), the concurrence quantifying two-qubit entanglement 54] is related to the spin squeezing parameter $\xi_{1}$ of Eq. (4) through $\mathcal{C}=1-\xi_{1}^{2}$, thereby implying the equivalence between spin squeezing and quantum entanglement. However, the squeezing parameter $\xi_{1}$ cannot apply to the non-symmetric state $\left|\Phi_{12}\right\rangle$ with $\beta \neq \gamma$ as the parameter can be larger than unity even for a non-symmetric product state. The spin squeezing criterion $\xi_{1}<1$ cannot separate an entangled state from a non-entangled one. The relationship between the local invariant spin squeezing parameter $\tilde{\xi}_{k}$ and the concurrence, for a pure state of two qubits given by Eq. (22), therefore provides a generalization of the earlier result [50] applicable to both symmetric as well as non-symmetric pure states of two qubits.

For the general two-qubit state we have

Proposition 2: Spin squeezing with local unitary invariance and quantum entanglement are equivalent for arbitrary two-qubit pure states. The quantitative relations between spin squeezing parameters and the concurrence are given by

$$
\tilde{\xi}_{1}=\sqrt{1-\mathcal{C}}, \quad \tilde{\xi}_{2}=\frac{1}{\sqrt{1+}} .
$$

Proof: Up to local unitary operations, we can express the state $\left|\Phi_{12}\right\rangle$ using a Schmidt decomposition through

$$
\begin{aligned}
& \left|\Phi_{12}\right\rangle=\lambda_{1}|00\rangle+\lambda_{2}|11\rangle, \\
& 0 \leq \lambda_{1}, \quad \lambda_{2} \leq 1, \lambda_{1}^{2}+\lambda_{2}^{2}=1,
\end{aligned}
$$

where the Schmidt coefficients $\lambda_{1}$ and $\lambda_{2}$ are related to $\alpha, \beta, \gamma$, and $\delta$ by

$$
\begin{aligned}
\lambda_{1}^{2} & =\frac{1}{2}\left[1+\sqrt{1+4|(\beta \gamma-\alpha \delta)|^{2}}\right], \\
\lambda_{2}^{2} & =\frac{1}{2}\left[1-\sqrt{1+4|(\beta \gamma-\alpha \delta)|^{2}}\right] .
\end{aligned}
$$

The mean spin is along the $z$ direction, and it is easy to see that

$$
\langle 0\rangle=\left|\lambda_{1}^{2}-\lambda_{2}^{2}\right| .
$$

Then, from Eq. (18), we find

$$
\begin{aligned}
\left(\Delta_{\left\{\theta_{i}\right\}}\right)^{2}= & \frac{1}{2}\left(1+\cos \theta_{1} \cos \theta_{2}\left\langle\sigma_{1 x} \sigma_{2 x}\right\rangle\right. \\
& +\sin \theta_{1} \sin \theta_{2}\left\langle\sigma_{1 y} \sigma_{2 y}\right\rangle \\
& +\sin \theta_{1} \cos \theta_{2}\left\langle\sigma_{1 y} \sigma_{2 x}\right\rangle \\
& \left.+\cos \theta_{1} \sin \theta_{2}\left\langle\sigma_{1 x} \sigma_{2 y}\right\rangle\right) \\
= & \frac{1}{2}\left[1+2 \cos \left(\theta_{1}+\theta_{2}\right) \lambda_{1} \lambda_{2}\right],
\end{aligned}
$$

which manifestly implies that

$$
\left(\Delta_{\left\{\theta_{i}\right\}}\right)_{\min }=\sqrt{\frac{1-2 \lambda_{1} \lambda_{2}}{2}} .
$$


Therefore, from Eqs. (25), (27), and the definition of squeezing parameters $\tilde{\xi}_{k}$, we have

$$
\tilde{\xi}_{1}=\sqrt{1-2 \lambda_{1} \lambda_{2}}, \quad \tilde{\xi}_{2}=\frac{\sqrt{1-2 \lambda_{1} \lambda_{2}}}{\left|\left(\lambda_{1}^{2}-\lambda_{2}^{2}\right)\right|} .
$$

Identifying now that the concurrence [54] is related to the Schmidt coefficents $\lambda_{1}, \lambda_{2}$ through

$$
=2 \lambda_{1} \lambda_{2}=2|(\beta \gamma-\alpha \delta)|,
$$

and expressing $\left|\left(\lambda_{1}^{2}-\lambda_{2}^{2}\right)\right|=\sqrt{1-{ }^{2}}$ in Eq. (28), we obtain the proposition.

This proposition reveals that a pair of qubits, which share a pure entangled state $(\neq 0)$, is always in a spin squeezed state. This one-to-one relation between spin squeezing and entanglement implies that we can use $\tilde{\xi}_{k}$ as a measure of entanglement for pure bipartite states of qubits. However, it should be noted that the maximum spin squeezing characterized by $\tilde{\xi}_{1}=0$ and $\tilde{\xi}_{2}=1 / \sqrt{2}$ for $=1$ (maximally entangled states) is realised in the limit of $\rightarrow 1$, since the qubits have no preferred orientation in space $\left(\left\langle\vec{\sigma}_{i}\right\rangle=0\right)$ when they share a maximally entangled state.

\section{B. Multi-qubit states}

We now examine the relations between spin squeezing and quantum entanglement for arbitrary multi-qubit states. For all separable $N$-qubit states, it was already shown that the original spin squeezing parameter $\xi_{2} \geq 1$ [29]. However, here the parameter $\tilde{\xi}_{2}$ is locally unitary invariant, and therefore more closely related to quantum entanglement as we have seen in Proposition 2.

For separable states, we have

Proposition 3: For all separable $N$-qubit states, $\tilde{\xi}_{2} \geq 1$. Proof: Along similar lines of proof $\xi_{2} \geq 1$ [29], we now give the proof. A separable state can be written as

$$
\rho=\sum_{k} p_{k} \rho_{1}^{(k)} \otimes \rho_{2}^{(k)} \otimes \ldots \otimes \rho_{N}^{(k)}, \quad \sum_{k} p_{k}=1 .
$$

Calculating the variance of $\left\{\theta_{i}\right\}$, we find

$$
\begin{aligned}
\left(\Delta_{\left\{\theta_{i}\right\}}\right)^{2}= & \frac{N}{4}+\frac{1}{4} \sum_{k} p_{k} \sum_{i=1}^{N} \sum_{j \neq i=1}^{N}\left\langle\vec{\sigma}_{i} \cdot \hat{n}_{\theta_{i}}\right\rangle_{k}\left\langle\vec{\sigma}_{j} \cdot \hat{n}_{\theta_{j}}\right\rangle_{k} \\
= & \frac{N}{4}+\frac{1}{4} \sum_{k} p_{k} \sum_{i} \sum_{j}\left\langle\vec{\sigma}_{i} \cdot \hat{n}_{\theta_{i}}\right\rangle_{k}\left\langle\vec{\sigma}_{j} \cdot \hat{n}_{\theta_{j}}\right\rangle_{k} \\
& -\frac{1}{4} \sum_{k} p_{k} \sum_{i}\left\langle\vec{\sigma}_{i} \cdot \hat{n}_{\theta_{i}}\right\rangle_{k}^{2} \\
= & \frac{N}{4}+\frac{1}{4} \sum_{k} p_{k}\left(\sum_{i}\left\langle\vec{\sigma}_{i} \cdot \hat{n}_{\theta_{i}}\right\rangle_{k}\right)^{2} \\
& -\frac{1}{4} \sum_{k} p_{k} \sum_{i}\left\langle\vec{\sigma}_{i} \cdot \hat{n}_{\theta_{i}}\right\rangle_{k}^{2} \\
\geq & -\frac{1}{4} \sum_{k} p_{k} \sum_{i}\left\langle\vec{\sigma}_{i} \cdot \hat{n}_{\theta_{i}}\right\rangle_{k}^{2} .
\end{aligned}
$$

Using the condition

$$
\left\langle\vec{\sigma}_{i} \cdot \hat{n}_{\theta_{i}}\right\rangle_{k}^{2}+\left\langle\vec{\sigma}_{i} \cdot \hat{n}_{\theta_{i}}^{\prime}\right\rangle_{k}^{2}+\left\langle\vec{\sigma}_{i} \cdot \hat{n}_{i 0}\right\rangle_{k}^{2} \leq 1
$$

with

$$
\hat{n}_{\theta_{i}}^{\prime}=-\sin \left(\theta_{i}\right) \hat{n}_{i \perp}+\cos \left(\theta_{i}\right) \hat{n}_{i \vdash}
$$

we obtain

$$
\begin{aligned}
& \frac{N}{4}-\frac{1}{4} \sum_{k} p_{k} \sum_{i}\left\langle\vec{\sigma}_{i} \cdot \hat{n}_{\theta_{i}}\right\rangle_{k}^{2} \\
\geq & \frac{1}{4} \sum_{k} p_{k} \sum_{i}\left(\left\langle\vec{\sigma}_{i} \cdot \hat{n}_{\theta_{i}}^{\prime}\right\rangle_{k}^{2}+\left\langle\vec{\sigma}_{i} \cdot \hat{n}_{i 0}\right\rangle_{k}^{2}\right) \\
\geq & \frac{1}{4} \sum_{k} p_{k} \sum_{i}\left\langle\vec{\sigma}_{i} \cdot \hat{n}_{i 0}\right\rangle_{k}^{2} \\
= & \frac{1}{N} \sum_{k, i}{\sqrt{p_{k}}}^{2} \sum_{k, i}\left(\sqrt{p_{k}} \frac{\left\langle\vec{\sigma}_{i} \cdot \hat{n}_{i 0}\right\rangle_{k}^{2}}{2}\right)^{2} \\
\geq & \frac{1}{4 N}\left(\sum_{k} p_{k} \sum_{i}\left\langle\vec{\sigma}_{i} \cdot \hat{n}_{i 0}\right\rangle_{k}\right)^{2} \\
= & \left.\frac{\langle 0}{N}\right\rangle^{2}
\end{aligned}
$$

where the last inequality follows from the CauchySchwarz inequality, and the last equality from

$$
\langle\rangle^{2}=\frac{1}{4}\left(\sum_{k} p_{k} \sum_{i}\left\langle\vec{\sigma}_{i} \cdot \hat{n}_{i 0}\right\rangle_{k}\right)^{2}
$$

in the separable state.

Therefore, we obtain the inequality

$$
\left(\Delta_{\left\{\theta_{i}\right\}}\right)^{2} \geq \frac{1}{N}\langle 0\rangle^{2},
$$

which leads to the result that $\tilde{\xi}_{2} \geq 1$ for all separable states.

In this way, local unitary invariant spin squeezing characterized by $\tilde{\xi}_{2}<1$ serves as a sufficient condition for quantum entanglement.

\section{CONCLUSION}

In conclusion, we have proposed a local invariant criterion of spin squeezing in multi-qubit systems, which is applicable for arbitrary multi-qubit states. Due to the local unitary invariant property of the spin squeezing criteria and quantum entanglement, we find that (i) for arbitrary two-qubit states, spin squeezing is equivalent to quantum entanglement, and (ii) for arbitrary multiqubit states, we find that the squeezing parameter $\tilde{\xi}_{2}<1$ implies quantum entanglement. The inequality $\tilde{\xi}_{2}<1$ serves as a sufficient condition for entanglement. 


\section{Acknowledgments}

ARU acknowledges the support of a Macquarie University Visiting Fellowship. We acknowledge valuable comments from Prof. Li You and helpful discussions with Do- minic W. Berry. This project has been supported by an Australian Research Council Large Grant and by an Australian Department of Education, Science and Training Innovation Access Program Grant to support the European Fifth Framework Project QUPRODIS.
[1] M.A. Nielsen and I.L. Chuang, Quantum Computation and Quantum Information, Cambridge University Press, 2000.

[2] D. Bouwmeester, A. Ekert, and A. Zeilinger, The Physics of Quantum Information: Quantum Cryptography, Quantum Teleportation, Quantum Computation, (Springer-Verlag, Berlin, 2000).

[3] C. H. Bennett, G. Brassard, C. Crépeau, R. Jozsa, A. Peres and W. K. Wootters, Phys. Rev. Lett. 70, 1895 (1993).

[4] A. Ekert, Phys. Rev. Lett. 67, 661 (1991).

[5] I. Cirac and N. Gisin, Phys. Lett. A 229, 1 (1997).

[6] C.A. Fuchs, N. Gisin, R.B. Griffiths, C.-S. Niu and A. Peres, Phys. Rev. A 56, 1163 (1997).

[7] S.L. Braunstein, C.M. Caves, R. Jozsa, N. Linden, S. Popescu and R. Schack, Phys. Rev. Lett. 83, 1054 (1999).

[8] A. Ekert and R. Jozsa, Philos. Trans. R. Soc., London Ser. A 356, 1769 (1998).

[9] C.H. Bennett and S. Wiesner, Phys. Rev. Lett. 69, 2881 (1992).

[10] J. Eisert, K. Jacobs, P. Papadopoulos and M.B. Plenio, Phys. Rev. A 62, 052317 (2000).

[11] J.I. Cirac, A. Ekert, S.F. Huelga and C. Macchiavello, Phys. Rev. A 59, 4249 (1999).

[12] A. Thapliyal, Phys. Rev. A 59, 3336 (1999).

[13] J. Kempe, Phys. Rev. A 60, 910 (1999).

[14] C.H. Bennett, D.P. DiVincenzo, T. Mor, P.W. Shor, J.A. Smolin and B.M. Terhal, Phys. Rev. Lett. 83, 3081 (1999).

[15] W. Dür, J.I. Cirac and R. Tarrach, Phys. Rev. Lett. 83, 3562 (1999).

[16] D.P. DiVincenzo, T. Mor, P.W. Shor, J.A. Smolin and B.M. Terhal, e-print quant-ph/9908070

[17] J.A. Smolin, e-print quant-ph/0001001

[18] W. Dür and J.I. Cirac, Phys. Rev. A 62, 022302 (2000).

[19] P.W. Shor, J.A. Smolin and A.V. Thapliyal, e-print quant-ph/0005117

[20] A. Wong and N. Christensen, Phys. Rev. A 63, 044301 (2001)

[21] D.J. Wineland, J.J. Bollinger, W.M. Itano, F.L. Moore and D.J. Heinzen, Phys. Rev. A 46 R6797 (1992)

[22] A. Kuzmich, K. Mølmer and E.S. Polzik, Phys. Rev. Lett. 79, 4782 (1997).

[23] A. Kuzmich, N.P. Bigelow and L. Mandel, Europhys. Lett. 42, 481 (1998).

[24] E.S. Polzik, Phys. Rev. A 59, 4202 (1999).

[25] J. Hald, J.L. Sørensen, C. Schori and E.S. Polzik, Phys. Rev. Lett. 83, 1319 (1999)

[26] A. Kuzmich, L. Mandel and N.P. Bigelow, Phys. Rev. Lett. 85, 1594 (2000).

[27] L.-M. Duan, J.I. Cirac, P. Zoller and E.S. Polzik, Phys. Rev. Lett. 85, 5643 (2000).

[28] A. Kozhenkin, K. Mølmer and E.S. Polzik, Phys. Rev. A 62, 033809 (2000).
[29] A. Sørensen, L.-M.Duan, J.I. Cirac, and P. Zoller, Nature (London), 409, 63 (2001).

[30] M. Kitagawa and M. Ueda, Phys. Rev. A 47, 5138 (1993).

[31] M.D. Lukin, S.F. Yelin, and M. Fleischhauer, Phys. Rev. Lett. 84, 4232 (2000); A. André and M.D. Lukin, Phys. Rev. A 65, 053819 (2002).

[32] L. Vernac, M. Pinard, and E. Giacobino, Phys. Rev. A 62, $063812(2000)$.

[33] K. Helmerson and L. You, Phys. Rev. Lett. 87, 170402 (2001); Ö. E. Müstecaplioğlu, M. Zhang, and L. You, Phys. Rev. A 66, 033611 (2002); S. Yi, Ö. E. Müstecaplioğlu, C.P. Sun, and L. You, ibid. 66, 011601 (2002); Ö. E. Müstecaplioğlu, M. Zhang, and L. You, ibid. 66, 033611 (2002).

[34] C. Orzel, A. K. Tuchman, M. L. Fenselau, M. Yasuda, and M. A. Kasevich, Science 291, 2386 (2001).

[35] U. Poulsen and K. Mølmer, Phys. Rev. A 64, 013616 (2001)

[36] D. W. Berry and B. C. Sanders, New J. Phys. 4, 8 (2002).

[37] L. K. Thomsen, S. Mancini, and H.M. Wiseman, Phys. Rev. A 65, 061801 (2002); D.W. Berry and B.C. Sanders, ibid. 66, 012313 (2002).

[38] A. R. Usha Devi, K. S. Mallesh, M. A. A. Sbaih, K.B. Nalini, and G. Ramachandran, J. Phys. A: Math. Gen. 36, 5333 (2003).

[39] T. Gasenzer, D. C. Roberts, and K. Burnett, Phys. Rev. A 65, 021605 (2002).

[40] J.K. Stockton, J.M. Geremia, A.C. Doherty, and H. Mabuchi, Phys. Rev. A 67, 022112 (2003).

[41] Z. Ficek and R. Tanaś, Phys. Rep. 372, 369 (2002).

[42] L. Zhou, H.S. Song and C. Li, J. Opt. B: Quantum Semiclass. Opt. 4, 425 (2002).

[43] X. Wang, A. Sørensen, and K. Mølmer, Phys. Rev. A 64, 053815 (2001); X. Wang, J. Opt. B: Quantum Semiclassical Opt. 3, 93 (2001); X. Wang, Opt. Commun. 200, 277 (2001).

[44] A. Sørensen, K. Mølmer, Phys. Rev. Lett. 86, 4431 (2001).

[45] K. Wódkiewicz, and J.H. Eberly, J. Opt. Soc. Am. B 2, 458 (1985).

[46] G. S. Agarwal, and R. R. Puri, Phys. Rev. A 413782 (1990) ; G.S. Agarwal, and R.R. Puri, ibid. 4968 (1994).

[47] D. J. Wineland, J. J. Bollinger, W.M. Itano and D.J. Heinzen, Phys. Rev. A 50, 67 (1994).

[48] B. Yurke, Phys. Rev. Lett. 56, 1515 (1986); M. Kitagawa, and M. Ueda, ibid. 67, 1852 (1991).

[49] R. Horodecki and M. Horodecki, Phys. Rev. A 54, 1838 (1996).

[50] D. Ulam-Orgikh and M. Kitagawa, Phys. Rev. A 64, 052106 (2001).

[51] D. W. Berry and B.C. Sanders, New J. Phys. 4, 8 (2002).

[52] M.G. Raymer, A.C. Funk, B.C. Sanders, and H. de Guise, Phys. Rev. A 67, 052104 (2003).

[53] X. Wang and B.C. Sanders, Phys. Rev. A 68, 0121XX 
(2003); X. Wang and K. Mølmer, Eur. Phys. J. D 18, 385 (2002).

[54] S. Hill and W.K. Wootters, Phys. Rev. Lett. 78, 5022

(1997); W.K. Wootters, ibid. 80, 2245 (1998). 